\title{
Analysis on Examination Form and Strategies of New HSK
}

\author{
Yuwei Qiao \\ Heihe University \\ Heihe, China
}

\author{
Chunxue Jiang \\ Harbin Normal University \\ Harbin, China
}

\author{
Zhichun Zhu \\ Heihe University \\ Heihe, China
}

\begin{abstract}
The article fully analyzes characteristics, structure and form of New HSK and emphasizes teachers and students shall pay high attention to New HSK Outline. Under this premise, it discusses strategies to tutor grammar, vocabulary, listening, reading and writing respectively.
\end{abstract}

Keywords-new HSK; examination form; content of examination; strategies

\section{INTRODUCTION}

HSK is one of the important means for the teaching of Chinese as a foreign language and plays a positive role in the work of "promoting Chinese language in the world and enhancing understanding of various countries in the world on China". However, in recent years, problems are exposed in the process of promotion and implementation. To maintain the authority of Chinese language testing in native country, expand the influence of HSK and make it better meet the requirements of international promotion of Chinese language, the reform of HSK shall be put on the agenda.

\section{FORMS OF NEW HSK}

Defined as standardized test of international Chinese language proficiency, new HSK is to examine the ability of learners of Chinese language whose mother tongue is not Chinese to use Chinese language to communicate. The examination is divided into written examination and oral examination. Candidates need to take them respectively. Written examination include six levels with difficulty from low to high; the oral examination includes elementary, intermediate and advanced and adopts the form that record in audio-visual classroom.

Candidates passing level 1 can use very simple words and sentences. It belongs to entry level to make students like Chinese language rapidly; candidates passing level 2 can use Chinese language to make simple communication in familiar scene; candidates passing level 3 can use Chinese language to finish basic communicative tasks, and they can communicate

Fund program: This article is the staged achievement of teaching reform project of higher education in Heilongjiang province, "Research on Bottleneck Problems and Countermeasures of Education for Foreign Students in Local Universities in Heilongjiang", project number: JG2013010440. basic information when travel in China; candidates passing level 4 can use Chinese language to discuss and communicate fluently; candidates passing level 5 can read newspapers and periodicals and use Chinese language to make complete speech candidates passing level 6 can easily know about the information in listening and reading and fluently express in oral and written forms.

\section{PRINCIPLES AND PURPOSES OF THE EXAMINATION}

According to requirements of the Ministry of Education, the examination shall follow the principle of "combination of examination and teaching", closely integrate design and arrangement of the examination with current situation of Chinese language teaching in the world and Chinese teaching materials at present, in order to let examination and teaching supplement each other. New HSK has correct rating and evaluation and examine the ability of candidates to use Chinese language, let students improve Chinese proficiency designedly and effectively.

The result of new HSK can meet requirements of many aspects: provide effective reference basis for universities to recruit students, give lessons through class grouping, open exempted courses and grant credits; provide correct reference basis for employing organizations to hire and train working staff; let learners of Chinese language know about their application level of Chinese; provide authoritative basis for teaching and training of Chinese.

\section{CURRENT SITUATION OF NEW HSK}

In order to meet the demand of international promotion of Chinese language, National Office for Teaching Chinese as a Foreign Language (NOCFL) introduced new HSK IN November 2009. In 2010, the new HSK was carried out for nine times in 174 testing centers in 63 countries of the world, excluding China. 98,291 people took the examination, with growth rate of $29.18 \%$. Because oral test is newly added, the candidates taking oral test account for small proportion. Most people taking the exam are under 35 years old. Female candidates account for more than $60 \%$. The number of learners of Chinese language who take the new HSK and come from 
South Korea is the most, accounting for a very large proportion. Few candidates taking new HSK come from Europe and America. We need to further intensify the promotion. Among six levels of written examination, the number of candidates taking level 5 is the most. There are also some candidates who take low levels such as level 1 and level 2. Although few candidates taking low levels, we have achieve the purpose of promoting new HSK, to enlarge popularization of Chinese language, let learners of Chinese language start easily, let more learners of Chinese language get the certificate at the beginning and encourage them to study further. On the whole, according to statistics of candidates of new HSK, it can be seen that the international promotion of Chinese language becomes better, bigger and faster with each passing day.

\section{CONTENTS OF NEW HSK}

\section{A. Listening}

Listening is the most important in language use. In examination, it examines whether candidates can make effective judgment on contents heard by them in the shortest period of time and understand as well as sublimate. It also checks vocabulary, ability to speak, comprehend, remember, knowledge background and learning experience of candidates.

\section{B. Reading}

Vocabulary and reading comprehension are contents of language testing all the time. Vocabulary is the basis of reading. The quantity and proficiency of vocabulary have direct relationship with level of reading comprehension. Standard and Outline of HSK Level formulated by China Association for the teaching of Chinese as a foreign language plays a guiding role in reading of HSK. In terms of the time occupied by reading in new HSK, level 1 accounts for 57\%; level 2 accounts for $50 \%$; level 3 accounts for $35 \%$; level 4 accounts for $40 \%$; level 5 accounts for $37.5 \%$; level 6 accounts for $37 \%$. The types of reading include gap filling, true or false questions, choice question, arrangement of sentences, finding out faulty wording or formulation, choosing sentence to fill in the blanks and paragraph reading with difficulty from low to high.

HSK is proficiency test, so it examines ability in universal sense. The testing materials shall be extensive and diversified, instead of coming from a certain field. It can come from magazines and newspapers, involving contents of culture and economy. The questions shall be common sense, so it can be effective and fair.

\section{Writing}

Level 1 and level 2 of new HSK do not include writing. Level 3 includes two parts: the first part is to complete sentences, namely correctly place words according to order of Chinese language; the second part is fill in Chinese characters according to Chinese pinyin and it mainly examines vocabulary of candidates. Level 4 has two parts: the first part is to complete sentences, namely correctly place words according to order of Chinese language; the second part is to make sentences according to pictures. It gives keywords and open-and-shut pictures, so candidates can make sentences according to requirements. Level 5 has two parts: the first part is to complete sentences, including declarative sentences and interrogative sentences; the second part is to write two short passages. One gives keywords and the other gives pictures. One part in level 6 is abbreviation. First, let candidates read an article within ten minutes without transcribing, and then the examiner takes back the article and asks candidates to retell the main content on answer sheet with no need of writing opinions of their own. It is very difficult for foreigners who get used to writing letters to write Chinese characters. At present, teachers mainly teach via Chinese pinyin. Students can write letters and mark the pronunciation similar to language of their own. It leads to the fact that students write letters in the whole article. It does not follow the principles of Chinese characters teaching at all. So in the writing, it is necessary to let candidates write Chinese characters clearly. It is the success of Chinese language teaching. The meaning in letting candidates write out is bigger than the meaning in letting them analyze and comprehend. Therefore, if students can recognize relevant vocabularies, they can do well in writing. It is not very difficult in writing in general. The main purpose is to let candidates write them out.

\section{THEORETICAL BASIS OF EXAMINATION}

Language testing is to deduce the target form through measuring representation behavior of target skill. It is the basic definition of language testing. Knowledge, skill or ability measured by examination is intangible. We can only measure them through visible performance showed by them. Here, we can introduce mathematical inference. If $\mathrm{B}$ is representation of A, B can deduce A. That is to say, students show B in learning language, we can deduce the degree reached by students in learning language, namely A. Of course, any examination cannot examine all representation behaviors of learning, but carry out sample testing in all possible representations. The sample must be effective and extensive enough, covering all relevant elements. For instance, students learn one hundred words, including nouns, verbs and pronouns, which shall include words with regular and irregular spelling and words that appear earlier and appear later. The sampling is not random but well-rounded.

\section{RELIABILITY OF EXAMINATION}

The reliability of examination means whether the result of examination is reliable, namely whether the scores can properly show performance of candidates. It directly shows whether the results of examination are just and fair and whether candidates answer the questions justly and fairly.

The reliability of an examination is influenced by many things, such as the quantity and quality of examination paper, implementation process of examination, consistency of marking examination papers after examination. It is important to keep the reliability just and fair. To guarantee reliability of the examination, we shall ensure following three aspects. It is impossible to realize $100 \%$ reliability of examination, but we shall guarantee it from each link. 
- Quantity and quality of test questions: The quantity means that the quantity of test questions shall be sufficient. Only sufficient quantity can guarantee coverage of test questions, reasonable coverage and avoid tricky questions; quality means the results of examination can be scattered and distinguish degree of learning of candidates. The difficulty of test questions shall be moderate and suitable for candidates, belonging to a certain scope. The structure, type as well as contents, scope and requirements of test questions shall be stable.

- The implementation of examination shall be fair as well. It shall ensure consistency for all candidates in the same examination room, on a national scale as well as around the world. It includes the consistency of test questions, record, rules of examination and invigilation behaviors. The marking criterion shall be consistent and stable. Scorers shall guarantee consistency in marking examination papers as well as keep consistency with other scorers. They shall follow the criterion.

\section{VALIDITY OF EXAMINATION}

Validity of examination does not mean efficiency but mean whether the examination is effective and reaches established goal and accuracy of evaluation results. The validity includes the several following kinds.

\section{A. Internal Validity}

It mainly checks whether the examination covers knowledge points prescribed by the outline. The main guarantee is that whether the requirements of outline are met strictly in the process of research and development of test questions; whether it bases on effective view of language. Here, it has no relationship with structure of test questions. It checks whether the principle theories of examination have validity.

\section{B. External Validity}

It refers to the validity obtained by verifying the standard system except for examination. The purpose is to check whether it can judge current situation of ability of candidates and whether it can predict development in the future. Experts of testing generally think that external standard is the most objective, and the validation method on the basis of external standard is the most scientific.

\section{Validity of Use}

It Shall Be Called Validity of Reaction of Candidates, and Reliability That Whether It Can Get The Identification From Candidates Who Take The Examination.

\section{Beyond the Test Validity:}

It refers to validity beyond purpose of the examination. Except for examining current ability of candidates, it means whether the examination can judge the candidates are competent for related work and bring effective working basis for candidates. Except for examination, it also needs good backwash validity to check whether it can stimulate enthusiasm of teachers and students to learn conversely.

In a word, the purpose of examination is to let students learn knowledge and strengthen the enthusiasm to learn Chinese language in the process of preparing for the examination. Because authoritative examination can let students get the most advanced and the most correct capability evaluation, instead of only telling the mark, the significance of examination is worthy of thinking of us. So far, we need to devote greater efforts to researching. Besides, we shall reinforce researching the relationship between outline and teaching materials to prevent that they depart from each other. It is important to let students get the evaluation or impetus after the examination. It needs to further discuss whether the evaluation is the most advanced and can best express the ability of candidates at present. After the examination, candidates only know they do not pass it instead of knowing the shortcomings. It needs time to better combine outline with teaching materials. It needs the support of NOCFL to help undergraduate and graduate in teaching Chinese as foreign language to work on related occupations. At present, domestic and foreign construction of teachers' team focuses on foreign language teachers and lack talents with education background and long-term cultivation. It still has a long way to go to keep these talents stay in this industry after graduation.

With increasing enhancement of national strengthen of our country, more and more people will be keen on Chinese culture and Chinese characters and learn Chinese language. As spreader of international Chinese language education, our country will devote to spread of Chinese language all the time. We need to spread HSK to every corner of the world to promote Chinese language, provide a solid and strong platform for beginners and fans who can speak Chinese fluently. The platform is HSK with reliability and validity.

\section{REFERENCES}

[1] Wu Jing, Preliminary Study on Composition Teaching of HSK, master's thesis in Jilin University, 2010.

[2] Ying Wei. Research on Bias Error in Noun of Locality in HSK Composition of Overseas Students from South Korea and Japan, Modern Language, 2010.

[3] Zhu Zhichun, Kong Yanmei. Research on Bottleneck Problems and Countermeasures of Education for Foreign Students in Local Universities in Heilongjiang [J], Intelligence, 2015(36). 